\title{
Study of MRS photodiodes for T2K experiment
}

Yu. Kudenko ${ }^{* a}$, E. Akhromeev ${ }^{a}$, G. Bondarenko ${ }^{b}$, V. Golovin ${ }^{b}$, E. Gushin ${ }^{a}$, A. Izmailov ${ }^{a}$, M. Khabibullin ${ }^{a}$, A. Khotjantsev ${ }^{a}$, B. Lubsandorzhiev ${ }^{a}$, O. Mineev ${ }^{a}$, Yu. Musienko ${ }^{a}$, A. Shaikhiev ${ }^{a}$, N. Yershov ${ }^{a}$

${ }^{a}$ Institute for Nuclear Research Russian Academy of Sciences

117312 Moscow, Russia

${ }^{b}$ Center of Perspective Technology and Apparatus, 107076 Moscow, Russia

E-mail: kudenko@inr.ru cpta@mail.transit.ru, izmaylov@inr.ru

Evgueni.Guschindcern.ch, marateinr.ru blexdinr.ru. plegeinr.ru.

lubsandepcbai10.inr.ruhep.rul Iouri.Moussienkodcern.ch,

shaykhieveinr.ru, yershoveinr.ru

Multi-pixel photodiodes operating in a limited Geiger mode will be used for photoreadout in the near detector ND280 of the long baseline neutrino oscillation experiment T2K. Basic parameters of MRS photodiodes such as the photon detection efficiency, gain, cross-talk, dark rate, stability, life-time, and others are discussed. Some results of study of Hamamatsu multipixel photon counters (MPPC's) are also presented. We discuss an application of MRS photodiodes for light readout from the side muon range detector (SMRD) composed of scintillator counters with WLS fibers.

International workshop on new photon-detectors PD07

June 27-29 2007

Kobe University, Kobe, Japan

* Speaker. 


\section{Introduction}

The T2K project [1] is a second generation long baseline neutrino oscillation experiment which will use a high intensity off-axis neutrino beam produced by the JPARC proton beam. The first phase of the T2K experiment pursues two main goals: a sensitive measurement of $\theta_{13}$ and a more accurate determination of the parameters $\sin ^{2} 2 \theta_{23}$ and $\Delta m_{23}^{2}$ than any previous experiment. To provide precise measurements of the neutrino beam properties, neutrino flux, spectrum and interaction cross sections, the near detector complex (ND280 [2]) will be built at a distance of $280 \mathrm{~m}$ from the target along the line between the average pion decay point and the Super-Kamiokande detector. The off-axis ND280 is shown in Fig. 1 and consists of the UA1 magnet operated with a magnetic

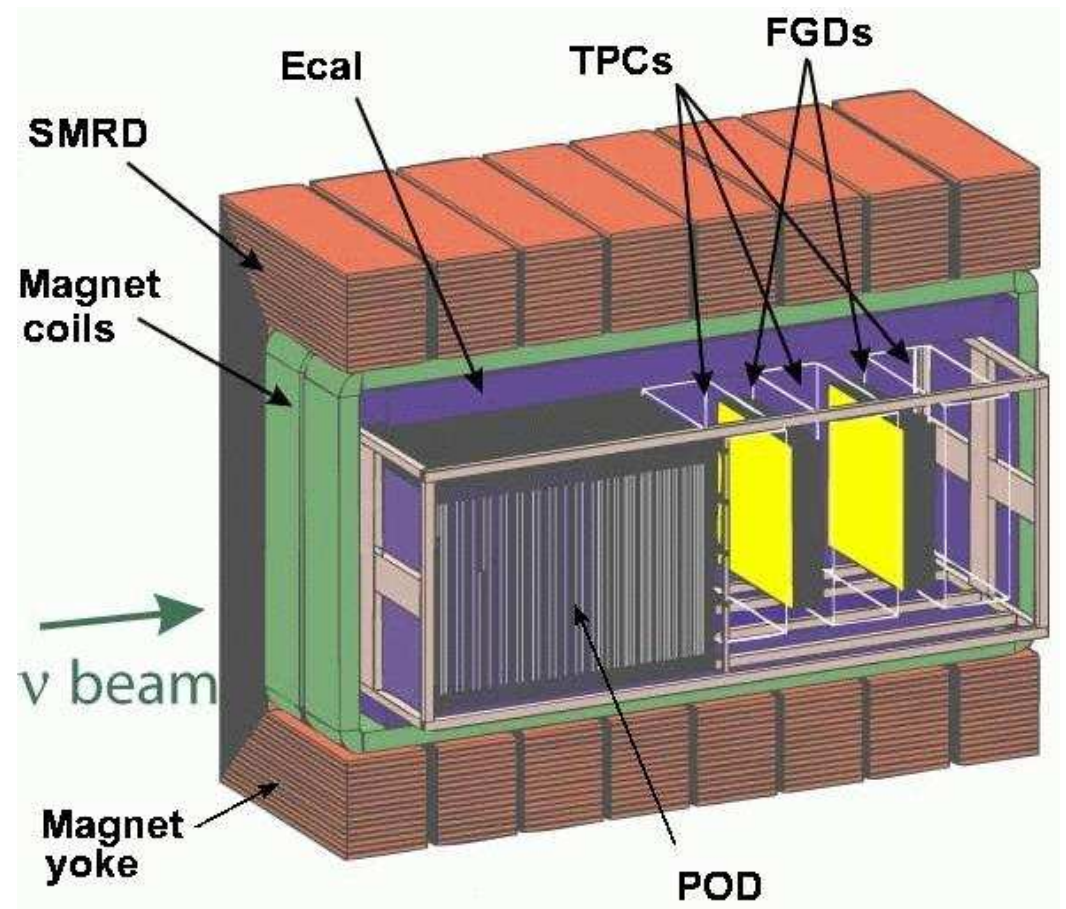

Figure 1: The cutaway view of the T2K near detector.

field of $0.2 \mathrm{~T}$, a Pi-Zero detector (POD), a tracking detector which includes time projection chambers (TPC's) and fine grained scintillator detectors (FGD's), an electromagnetic calorimeter (Ecal), and a side muon range detector (SMRD).

The ND280 detector will widely use wavelength shifting (WLS) fiber readout with light detection from fibers by photosensors which have to operate in a magnetic field environment and a limited space inside the UA1 magnet. The primary candidate for the photosensor is a multi-pixel avalanche photo-diode operating in the limited Geiger multiplication mode. Such photodiodes are compact, well matched to spectral emission of WLS fibers, and insensitive to magnetic fields [3, ฤ]. The required parameters for photosensors from all ND280 subdetectors can be summarized as follows. Diameter of an active area $\sim 1 \mathrm{~mm}$, high photon detection efficiency $(\geq 15-20 \%)$ for green light, number of pixels $>400$, the dark rate at operating conditions $\sim 1 \mathrm{MHz}$, gain should be around $(0.5-1.0) \times 10^{6}$, cross - talk $\sim 10 \%$, pulse width should be less than 100 ns to meet the 


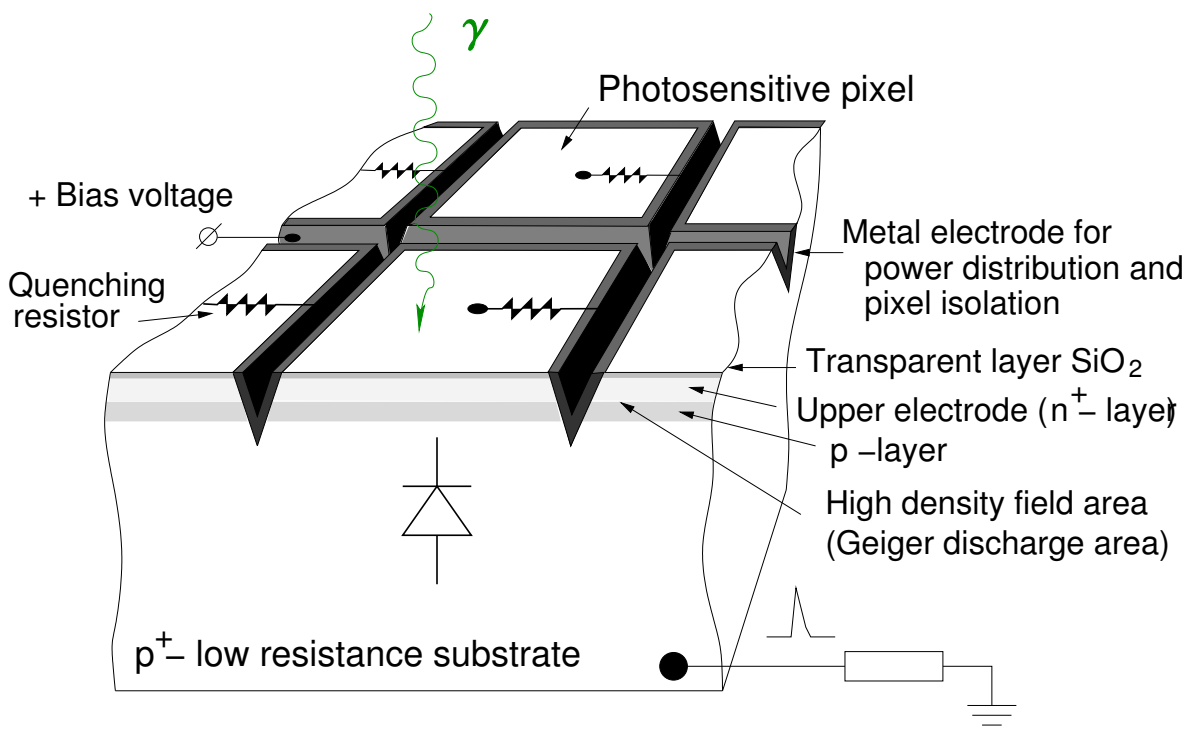

Figure 2: The schematic view of the MRS photodiode structure.

spill structure of the JPARC proton beam, readout electronics and the T2K triggers. For calibration and control purposes, it is very desirable to have well separated single photoelectron peaks at room temperature.

\section{Parameters of MRS photodiodes}

Detailed description of multi-pixel avalanche photodiodes with a metal-resistor-semiconductor layer structure operating in the limited Geiger mode (hereafter referred to as MRS APD's or MRS photodiodes) invented and designed by the Center of Perspective Technologies and Apparatus (CPTA), Moscow, can be found in Refs. [5, 6]. Such a photosensor consists of many pixels on a common $\mathrm{p}$-type silicon substrate. A simplified topology of a MRS photodiode is shown in Fig. 2. Each pixel operates as an independent Geiger micro-counter with a gain of the same order as a vacuum photomultiplier. Geiger discharge is initiated by a photoelectron in the locally high electric field created in a very thin layer $(\sim 1 \mu \mathrm{m}$ p-n junction) by the applied bias voltage. Small pixels are separated by grooves filled with an optically nontransparent material to suppress an optical cross-talk. The MRS photodiodes with a sensitive area of $1.1 \mathrm{~mm}^{2}$ with 556 pixels of $35 \times 35 \mu \mathrm{m}^{2}$ size of active area were manufactured. We have studied three types of such photodiodes: one operating at the bias voltage of about 42 volts $(42 \mathrm{~V})$, and two modified types $23 \mathrm{~V}$ and $30 \mathrm{~V}$ with increased sensitivity to green light. The main parameters of tested photodiodes are presented below.

The photon detection efficiency (PDE) of a multi-pixel avalanche photodiode operated in the limited Geiger mode is a product of 3 factors:

$$
\mathrm{PDE}=Q E \cdot \varepsilon_{\text {Geiger }} \cdot \varepsilon_{\text {pixel }},
$$

where $Q E$ is the wavelength dependent quantum efficiency, $\varepsilon_{\text {Geiger }}$ is the probability to initiate the Geiger discharge by a photoelectron, and $\varepsilon_{\text {pixel }}$ is a fraction of the total photodiode area occupied by 


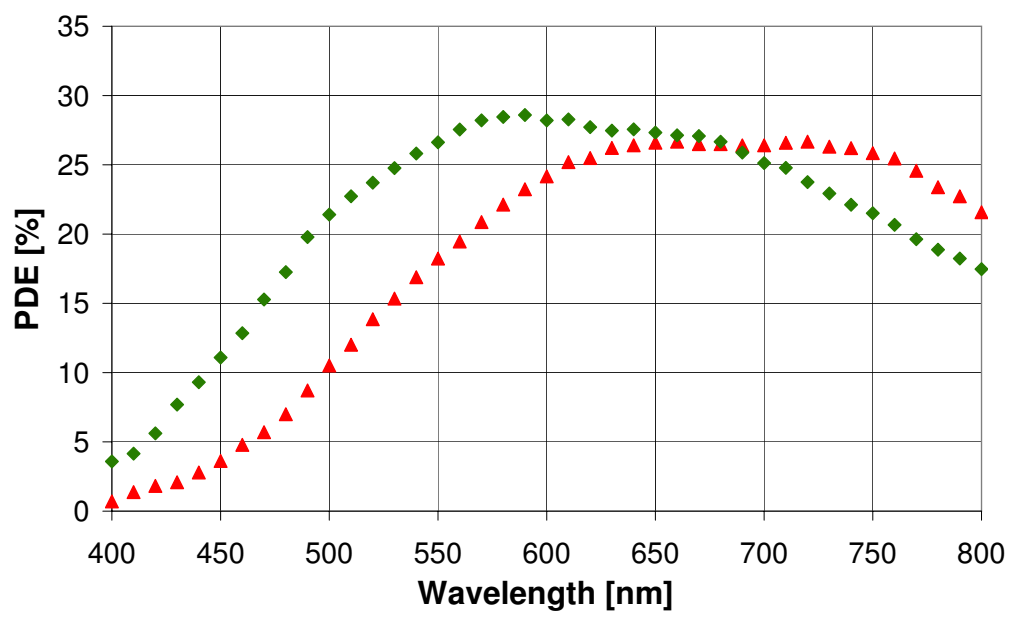

Figure 3: Photon detection efficiency of MRS photodiodes (red - 42V, black - 23V and 30V devices ) as a function of wavelength.

sensitive pixels. The bias voltage affects one parameter in expression (2.1), $\varepsilon_{\text {Geiger }}$. The geometrical factor $\varepsilon_{\text {pixel }}$ is completely determined by the MRS photodiode topology, and is estimated to be close to $70 \%$. The spectral responses of MRS photodiodes were measured in a spectrophotometer calibrated with a PIN-diode [7]. The absolute value of the PDE was measured using small pulses from a green LED $(525 \mathrm{~nm})$, which illuminated MRS photodiodes through a $0.5 \mathrm{~mm}$ diameter collimator. The number of emitted photons was obtained using a calibrated PMT XP2020. The operating bias voltage was tuned in all tested diodes to keep the dark rate of about $1 \mathrm{MHz}$ at a threshold of 0.5.p.e. The PDE dependence on the wavelength of the detected light for three types of the MRS APD's are shown in Fig. 3. Sensitivity to green light $(\lambda \sim 525 \mathrm{~nm})$ in both last modifications was increased by a factor of 2 in respect to $42 \mathrm{~V}$ devices. The cross-talk of all these devices was found to be about $5 \%$ at the expected operating bias voltage. The parameters of the MRS photodiodes were measured over temperatures from $15^{\circ} \mathrm{C}$ to $33^{\circ} \mathrm{C}$. The signal dependency of $-1.5 \% /{ }^{\circ} \mathrm{C}$ is obtained for increasing temperature, and in this temperature interval, the dark rate linearly depends on temperature with a coefficient of $62 \mathrm{kHz} /{ }^{\circ} \mathrm{C}[8]$.

Although the PDE of modified sensors was essentially increased, two parameters of these devices did not meet the requirements of the ND280. First, the gain of $23 \mathrm{~V}$ and $42 \mathrm{~V}$ devices is about 2 times less than that of the $42 \mathrm{~V}$ photodiode. Second, the pulse shape has a long tail of a few $\mu \mathrm{s}(23 \mathrm{~V})$ and shorter tail of about $1 \mu \mathrm{s}$ in the case of $30 \mathrm{~V}$ device. Typical pulse shapes of $42 \mathrm{~V}$ and $23 \mathrm{~V}$ photodiodes are shown in Fig 9 . This long tail cannot be easily accommodated to the beam structure and electronics readout, and an additional preamplifier/shaper is needed. The main parameters of tested MRS APD's are presented in Table 1 .

We also tested 10 samples of 400 pixel MPPC's. The description of these devices and the 

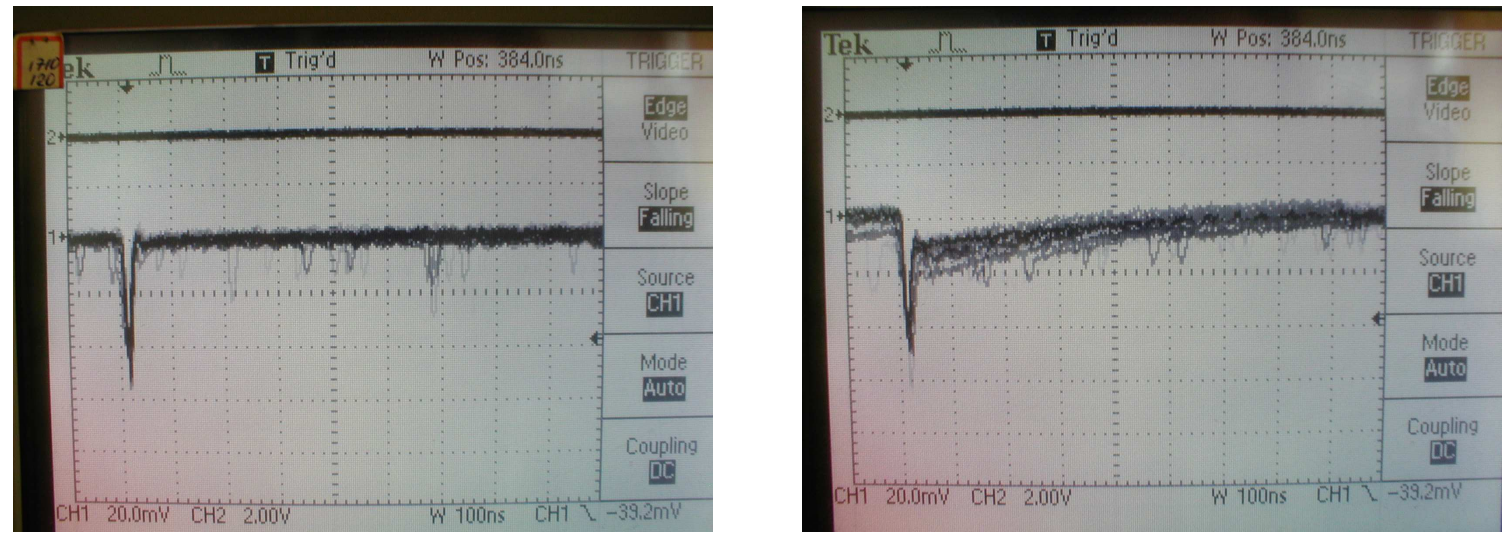

Figure 4: Signal amplitude vs time from two MRS photodiodes observed with a Tektronix TDS 2022 oscilloscope, at $20 \mathrm{mV}$ and $100 \mathrm{~ns}$ per division: $42 \mathrm{~V}$ device (left), and $30 \mathrm{~V}$ device (right).

\begin{tabular}{|l|ccc|}
\hline Parameter & $42 \mathrm{~V}$ & $23 \mathrm{~V}$ & $30 \mathrm{~V}$ \\
\hline Gain $\times 10^{6}$ & $0.5-0.7$ & $0.2-0.3$ & $0.3-0.4$ \\
$\mathrm{PDE}($ at $\lambda \sim 525 \mathrm{~nm}), \%$ & $10-12$ & $25-32$ & $24-30$ \\
Cross talk, $\%$ & $5-7$ & $5-7$ & $5-7$ \\
Pulse width & $<100 \mathrm{~ns}$ & tail a few $\mu \mathrm{s}$ & tail $\sim 1 \mu \mathrm{s}$ \\
\hline
\end{tabular}

Table 1: Main parameters of MRS photodiodes studied for SMRD counters

results of measurements can be found in Refs. [9, 10] The studied photodiodes demonstrated good performance: a low cross-talk value of about $10 \%$, a photon detection efficiency for green light of $\geq 12 \%$, a high gain of about $0.7 \times 10^{6}$, a low dark rate of $\sim 0.3 \mathrm{kHz}$ at operating voltage, and a pulse width of less than $50 \mathrm{~ns}$. The only potential problem (if afterpulses is not an issue [11]) is a high sensitivity of MPPC parameters to the ambient temperature. Fig. 5 the temperature variation of the MPPC signals from a green LED as a light source. The MPPC signal dependency of -(6-7) $\% /{ }^{\circ} \mathrm{C}$ is obtained from the measurements of six devices.

\section{SMRD counters with MRS photodiodes}

For large scintillator slabs used to instrument the UA1 magnet (SMRD) we employ a single WLS fiber embedded in a serpentine shaped (S-shape) groove. Scintillator slabs of $1 \times 17 \times$ $87 \mathrm{~cm}^{3}$ with the $\mathrm{S}$-shaped groove of $3 \mathrm{~mm}$ depth were manufactured at the Uniplast Factory, Vladimir, Russia. The scintillator was etched by a chemical agent that resulted in the formation of a micropore deposit over the plastic surface. The thickness of the deposit $(30-100 \mu \mathrm{m})$ depends on the etching time. Details can be found in Ref. [12]. The half-period of the $\mathrm{S}$-shape groove is $58 \mathrm{~mm}$, as shown in Fig. 6. A $2.55 \mathrm{~m}$ long multi-clad Kuraray Y11(200) WLS fiber of $1 \mathrm{~mm}$ diameter was embedded into the groove with an optical grease and both ends were attached to 42V MRS photodiodes. Typical ADC spectra for minimum ionizing particles obtained at two bias voltages are shown in Fig. 7. Well separated peaks are observed up to 12 photoelectrons. The WLS fiber route is clearly reflected in the pattern of the 1.y. distribution along the $\mathrm{x}$-axis, as seen in 


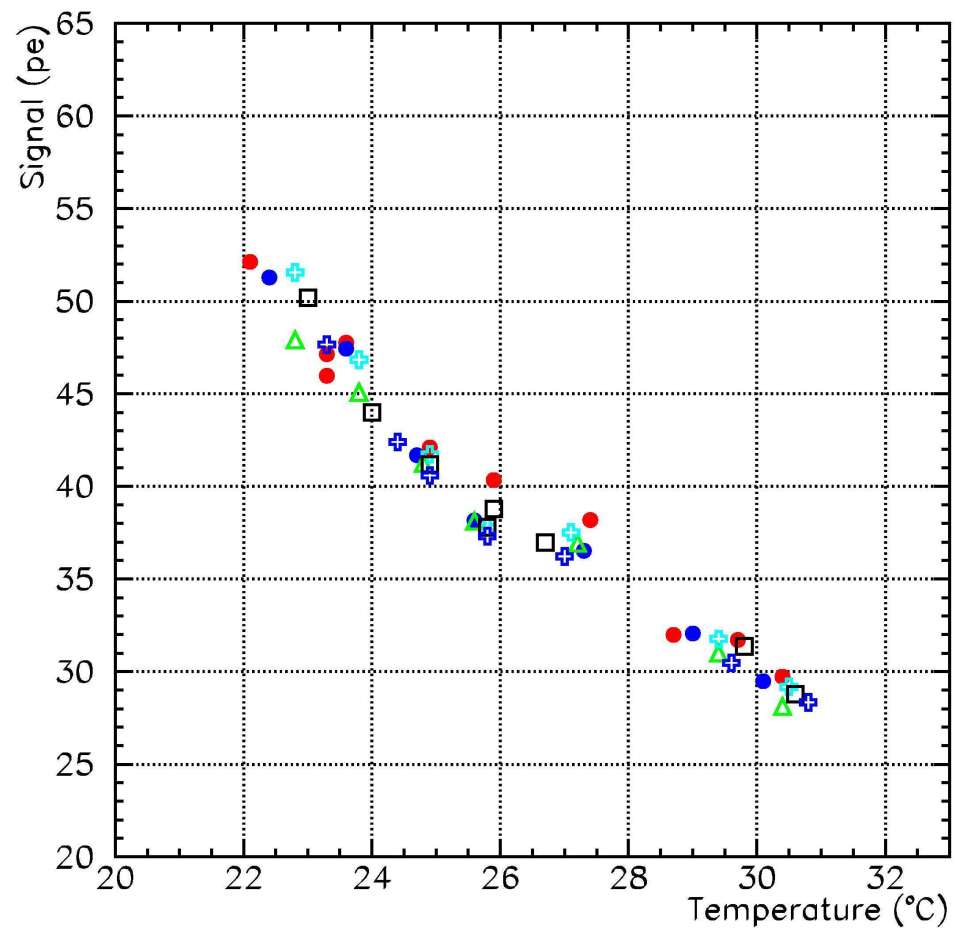

Figure 5: The signal from the MPPC photodiodes (6 devices) illuminated by a green LED as a function of the ambient air temperature.

Fig. 8. A maximum 1.y. was observed in points where the beam crossed the fiber, while the 1.y. dropped by about $20 \%$ in between the fiber segments. The light yield (sum of both end signals) was found to vary from 12 to 20 p.e./MIP over the S-counter surface that provides the MIP detection efficiency of greater than $99 \%$. The time resolution was measured at the discriminator thresholds set to 0.5 p.e. for each MRS photodiode. To suppress the time jitter caused by the trigger counters we used the time difference between two MRS signals: $\left(T_{\text {left }}-T_{\text {right }}\right) / 2$. The dependence of the time resolution on the light yield is presented in Fig. 9. The time resolution depends on photostatistics and is proportional to $1 / \sqrt{N_{p e}}$. At a typical 1.y. of 15 p.e./MIP, $\sigma_{t}$ is found to be $1.75 \mathrm{~ns}$, and it is mainly determined by the slow decay time of the Y11 fiber.

The usage of $23 \mathrm{~V}$ and $30 \mathrm{~V}$ devices allowed us to significantly increase the light yield of the SMRD counters. For example, for thinner SMRD S-counters ( $7 \mathrm{~mm}$ thickness of the scintillator) the average light yield of 20-23 p.e. was obtained at room temperature.

\section{Conclusion}

The performance of 3 types of MRS photodiodes designed for detection of green light was studied. $42 \mathrm{~V}$ devices have all parameters which satisfy the requirements of $\mathrm{T} 2 \mathrm{~K}$, but their PDE is relatively low. The PDE of MRS APD's operating at 23 and 30 volts is significantly higher, but pulse shape has a long tail that does not fit the proton beam structure. It is expected that the next 


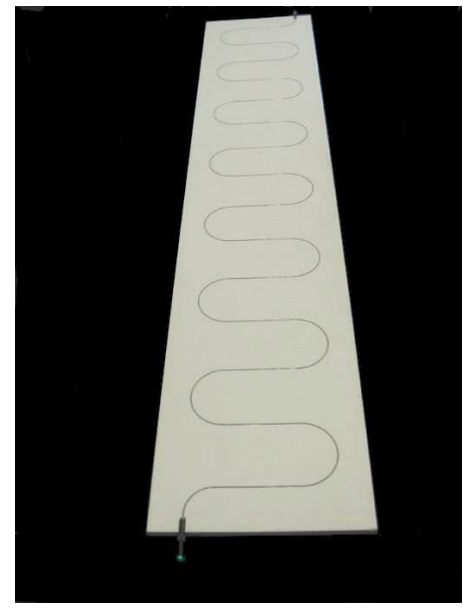

Figure 6: Scintillator slab with $\mathrm{S}$-shaped WLS fiber readout ( $\mathrm{S}-$ counter).

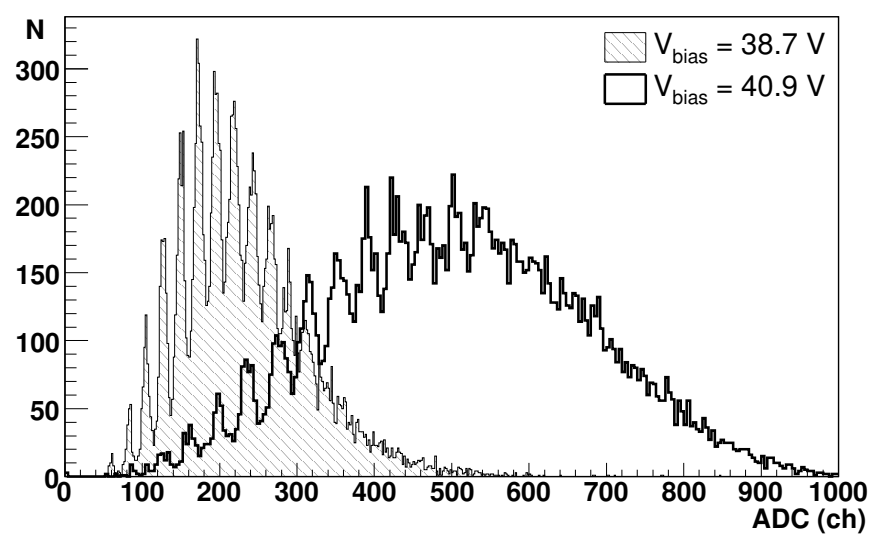

Figure 7: ADC spectra from minimum ionizing particles measured by the scintillator slab with the $42 \mathrm{~V}$ MRS APD at two bias voltages show clear peaks up to 12 photoelectrons.

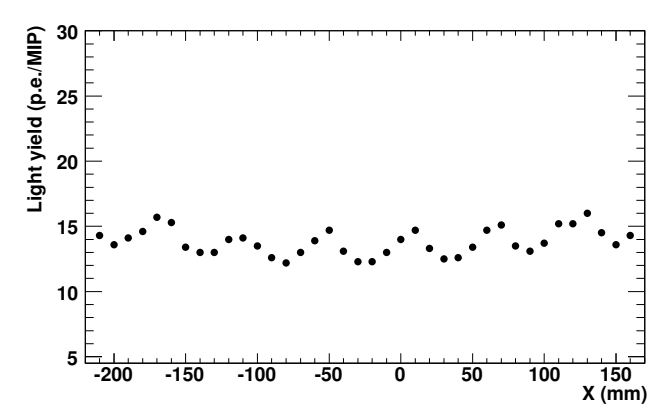

Figure 8: The light yield scan along the $\mathrm{S}$-counter. The oscillatory shape corresponds to the WLS fiber route with $58 \mathrm{~mm}$ spacing between neighboring segments.

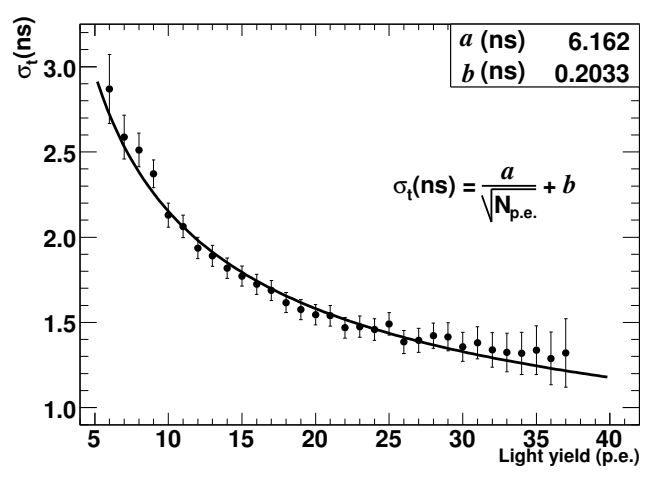

Figure 9: Time resolution vs light yield at the center of the $\mathrm{S}$-counter.

version of MRS photodiodes will have a high PDE and gain, and signal width less than $100 \mathrm{~ns}$ without a long tail. The MPPC's are met the T2K requirements if the ambient temperature is well controlled. The intensive studies of the life time and long term stability of these new devices, MRS APD's and MPPC's, have recently begun.

This work was supported in part by the "Neutrino Physics" Programme of the Russian Academy of Sciences.

\section{References}

[1] Y. Itow et al., hep-ex/0106019.

[2] “T2K ND280 Conceptual Design Report”, T2K Internal Document. 
[3] V. Andreev et al., Nucl. Instr. Meth. A540 (2005) 368.

[4] D. Beznosko et al., FERMILAB-PUB-05-410.

[5] V. Golovin et.al., Patent for invention in Russia, No. 1644708, 1989; A.G. Gasanov et al., Lett. J. Techn. Phys. 16 (1990) 14 (in Russian).

[6] G. Bondarenko et al., Nucl. Instr. Meth., A442 (2000) 187.

[7] E. Gushchin et al., Nucl. Instr. Meth. A567 (2006) 250.

[8] O. Mineev et.al., Nucl. Instrum. Meth. A577 (2007) 540 [arXiv:physics/0606037].

[9] Hamamatsu Photonics K. K., http://www.hamamatsu.com.

[10] M.Yokoyama et al., arXiv:physics/0606037.

[11] H. Oide, Study of afterpulsing of MPPC with waveform analysis, talk at this Workshop; F.Retiere, Using MPPC's for T2K fine grained detectors, talk at this Workshop.

[12] Yu.G. Kudenko et al., Nucl. Instr. Meth. A469 (2001) 340; O. Mineev et al., Nucl. Instr. Meth. A494 (2002) 362; N. Yershov et al., Nucl. Instr. Meth. A543 (2005) 454. 